\title{
Flows and bisections in cubic graphs
}

\author{
L. Esperet ${ }^{*} \quad$ G. Mazzuoccolo ${ }^{\dagger}$ M. Tarsi ${ }^{\ddagger}$
}

December 2, 2016

\begin{abstract}
A $k$-weak bisection of a cubic graph $G$ is a partition of the vertex-set of $G$ into two parts $V_{1}$ and $V_{2}$ of equal size, such that each connected component of the subgraph of $G$ induced by $V_{i}(i=1,2)$ is a tree of at most $k-2$ vertices. This notion can be viewed as a relaxed version of nowhere-zero flows, as it directly follows from old results of Jaeger that every cubic graph $G$ with a circular nowhere-zero $r$-flow has a $\lfloor r\rfloor$-weak bisection. In this paper we study problems related to the existence of $k$-weak bisections. We believe that every cubic graph which has a perfect matching, other than the Petersen graph, admits a 4 -weak bisection and we present a family of cubic graphs with no perfect matching which do not admit such a bisection. The main result of this article is that every cubic graph admits a 5-weak bisection. When restricted to bridgeless graphs, that result would be a consequence of the assertion of the 5-flow Conjecture and as such it can be considered a (very small) step toward proving that assertion. However, the harder part of our proof focuses on graphs which do contain bridges.
\end{abstract}

${ }^{*}$ Laboratoire G-SCOP (Univ. Grenoble Alpes, CNRS), Grenoble, France. Partially supported by ANR Project STINT (ANR-13-BS02-0007) and LabEx PERSYVAL-Lab (ANR-11-LABX-0025).

†Department of Computer Science, University of Verona, Italy. E-mail: giuseppe.mazzuoccolo@univr.it

$\ddagger$ The Blavatnik School of Computer Science, Tel Aviv University, Israel. E-mail: tarsi@post.tau.ac.il 


\section{Strong and weak bisections and how they relate to nowhere-zero flows}

This introductory section is mostly a brief reformulation and adaptation of some results stated and proved in [6] in terms of integer nowhere-zero flows and then in [9] in the more general setting of real valued flows.

Definition 1. Given a real number $r \geq 2$, a circular nowhere-zero $r$-flow ( $r$-CNZF for short) in a graph $G=(V, E)$ is an assignment $f: E \rightarrow[1, r-1]$ and an orientation $D$ of $G$, such that $f$ is a flow in $D$. That is, for every vertex $x \in V, \sum_{e \in E^{+}(x)} f(e)=\sum_{e \in E^{-}(x)} f(e)$, where $E^{+}(x)$, respectively $E^{-}(x)$, are the sets of edges directed from, respectively towards $x$ in $D$.

Accordingly defined is:

Definition 2. The circular flow number $\phi_{c}(G)$ of a graph $G$ is the infimum of the set of real numbers $r$, for which $G$ admits an $r$-CNZF. If $G$ has a bridge then we define $\phi_{c}(G)=\infty$.

The notion of $r$-CNZF was first introduced in [5], while observing that $(k, d)$-coloring, previously studied by Bondy and Hell [3], can be interpreted as the dual of a real (rather than integer)-valued nowhere-zero flow. The existence of $\phi_{c}(G)$ and the fact that it is always rational, was also observed and stated in [5]. Integer nowhere-zero flows are much more widely known and intensively studied, since first presented by W. Tutte [11, 60 years ago.

Along the article we use the following notational conventions: Let $G=$ $(V, E)$ be a graph.

- Given a set of vertices $X \subseteq V$, the set of edges with one endvertex in $X$ and the other one in $V \backslash X$, known as the edge-cut induced by $X$, is denoted here by $E(X)$.

- A bridge is an edge-cut which consists of a single edge. A graph with no bridge is bridgeless.

- When $D$ is an orientation of $G, E(X)$ is partitioned into $E^{+}(X)$ consisting of the edges directed from $X$ to $V \backslash X$, and $E^{-}(X)$, the set of edges directed from $V \backslash X$ to $X$. 
Definition 3. A bisection of a cubic graph $G=(V, E)$ is a partition of its vertex set $V$ into two disjoint subsets $V_{1}$ and $V_{2}$ of the same cardinality $\left|V_{1}\right|=\left|V_{2}\right|$.

For reasons clarified in the sequel we also define:

Definition 4. Let $k \geq 3$ be an integer. A bisection $\left(V_{1}, V_{2}\right)$ of a cubic graph $G=(V, E)$ is a $k$-weak bisection if every connected component of each of the two subgraphs of $G$, induced by $V_{1}$ and by $V_{2}$ is a tree on at most $k-2$ vertices. Such a component is referred to in the sequel as monochromatic.

Let $D$ be an orientation of a graph $G=(V, E)$ and let $f$ be an $r$-CNZF in $D$. Then for any $x \in V$, both $E^{+}(\{x\})$, and $E^{-}(\{x\})$ are non-empty. If $G$ is cubic then $V$ is partitioned into two sets $V_{1}, V_{2}$, where $V_{i}$ consists of all vertices of out-degree $i$ in $D$. That partition is clearly a bisection.

Furthermore, assume that there is a monochromatic path $x y z$ in $V_{1}$. Counting the relevant ingoing and outgoing edges reveals $E^{+}(\{x, y, z\})=1$ and $E^{-}(\{x, y, z\})=4$, and then it follows from the definition of an $r$-CNZF that $r \geq(4 / 1)+1=5$. If $V_{1}$ contains a monochromatic cycle, then all the edges with exactly one endpoint in this cycle are directed toward the cycle in $D$, which contradicts the fact that $D$ has an $r$-CNZF (regardless of the value of $r$ ). Clearly $V_{1}$ can be replaced here by $V_{2}$. Hence, we have shown that any cubic graph $G$ with $\phi_{c}(G)<5$ has a 4 -weak bisection. The same degree counting arguments leads to the following general observation, explicitly stated and proved (for integer values of $r$ ) in [6] :

Proposition 5. If a cubic graph $G$ admits an $r$-CNZF then there exists a $\lfloor r\rfloor$-weak bisection of $G(\lfloor r\rfloor$ is the largest integer, smaller or equal to $r)$.

Also stated and proved in [6] and [9] is the following stronger theorem, which fully characterizes cubic graphs (an instance of a more general statement which refers to all graphs) which admit $r$-CNZFs, in terms of bisections:

Theorem 6. Let $r \geq 3$ be a real number. A cubic graph $G=(V, E)$ admits an $r$-CNZF if and only if there exists a bisection $\left(V_{1}, V_{2}\right)$ of $G$, such that for every set of vertices $X \subseteq V$,

$$
|E(X)| \geq \frac{r}{r-2}|| V_{1} \cap X|-| V_{2} \cap X||
$$


The "only if" part of Theorem 6 is proved by applying the same counting argument as above to all subsets $X$ of $V$, while the proof of Proposition 5 only considers the subsets $X$ of $V_{1}$ and $V_{2}$. The "if" part also requires some basic flow network theory.

We refer to a bisection which satisfies the condition of Theorem 6 as an $r$-strong bisection of $G$. Note that an $r$-strong bisection is clearly also a $\lfloor r\rfloor$-weak bisection.

By definition, a cubic graph $G$ admits a 3-weak bisection if and only if it is bipartite, in which case that bisection $\left(V_{1}, V_{2}\right)$ consists of the two "sides" of the bipartition of $V$. It is easy to observe that $\left(V_{1}, V_{2}\right)$ in that case is also a 3-strong bisection. Proposition 5 hence implies that the circular flow number $\phi_{c}(G)$ of a cubic graph $G$ is never strictly between 3 and 4 .

\section{On 4-weak bisections}

It had been suggested by Amir Ban and Nati Linial [1] that every cubic graph other than the Petersen graph, admits a 4-weak bisection (where every monochromatic component is either an isolated vertex or a single edge). However, we later found an infinite family of counterexamples to that statement. These examples, hereby presented, are also quoted in [2].

A frequently used module in our construction is the graph $L_{k}(k \geq 0)$ depicted in Figure 1. Note that $L_{0}$ is obtained from $K_{3,3}$ by the removal of one edge.

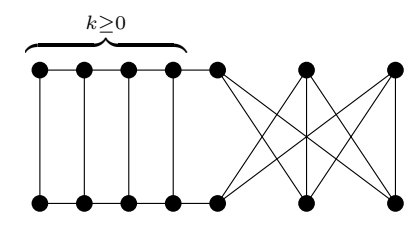

Figure 1: $L_{k}$

We say that a 2-coloring of the vertices of a graph (not necessarily cubic) is balanced if the two color classes are of the same size.

Lemma 7. For any $k \geq 0$, any 2-coloring of $L_{k}$ with no monochromatic component on more than 2 vertices is balanced and the two vertices of degree 2 are of distinct colors. 
Proof. We prove the result by induction on $k$. The two vertices of degree 2 are denoted by $u$ and $v$. When referring to the color of a vertex we relate to any given 2-coloring of $L_{k}$ with no monochromatic component of size 3 or more.

Assume first that $k=0$. If two adjacent vertices have the same color, say 1 , then all four remaining vertices have to be colored 2 and some vertex colored 2 has two neighbors colored 2 as well, which is a contradiction. It follows that without loss of generality the two neighbors of $u$ (resp. $v$ ) are colored 1 (resp. 2). As a consequence, $u$ and $v$ are colored 1 and 2, respectively and the coloring is indeed balanced.

If $k \geq 1$, then by removing $u$ and $v$ we obtain $L_{k-1}$. By the induction hypothesis, the coloring of the copy of $L_{k-1}$ is balanced and the two vertices of degree 2 of the copy of $L_{k-1}$ have distinct colors. It follows that if $u$ and $v$ have the same color, then one of them has the same color as its two neighbors, which is a contradiction. Therefore, $u$ and $v$ have distinct colors, and the coloring is balanced.

For any $k \geq 0$, let $L_{k}^{\prime}$ be the graph obtained from $L_{k}$ by adding a vertex adjacent to the two vertices of degree 2 of $L_{k}$, and let $T_{k}$ be the graph obtained by taking one copy of $L_{k}^{\prime}$ and two copies of $L_{0}^{\prime}$ and adding a new vertex adjacent to the three vertices of degree 2 (see Figure 2).

Proposition 8. For any $k \geq 0$, the graph $T_{k}$ has no 4-weak bisection.

Proof. Let $x$ be the vertex whose neighbors are the vertices of degree two in the copies of $L_{k}^{\prime}$ and $L_{0}^{\prime}$. Consider an arbitrary 4-weak bisection of $T_{k}$. Note that by Lemma 7, the corresponding 2-colorings of the copies of $L_{k}$ and $L_{0}$ are balanced, and the color of $x$ has to be distinct from the colors of its three neighbors. Therefore, the sizes of the two color classes differ by 2 .

The two copies of $L_{0}$ can clearly be replaced by one copy of $L_{i}$ and one of $L_{j}$, for any $i, j \geq 0$.

It so happens that each of our counterexamples contains a bridge. Furthermore, all these examples lack a perfect matching, and, as far as we can tell, that may be true for every counterexample. We therefore rephrase Ban and Linial's idea as follows:

Conjecture 9. The Petersen graph is the only cubic graph which admits a perfect matching, but no 4-weak bisection. 


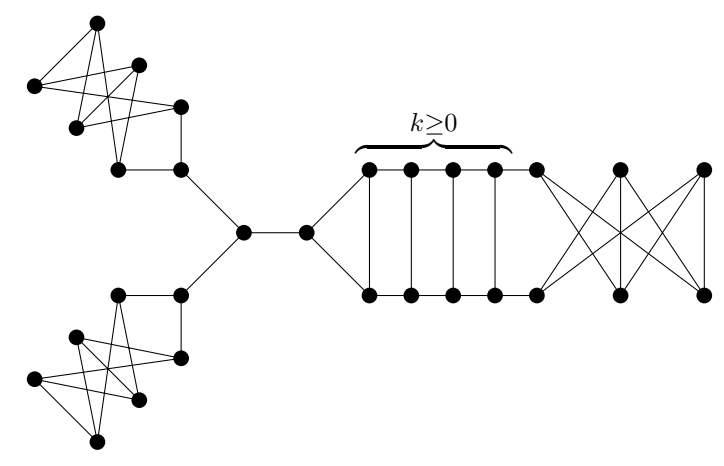

Figure 2: $T_{k}$, an infinite family of cubic graph with no 4 -weak bisections

A slightly weaker version of Conjecture 9, referring to bridgeless cubic graphs (which do admit perfect matchings [7]) is included in [2].

As a result of Proposition 5 , every cubic graph $G$ with $4 \leq \phi_{c}(G)<5$ admits a 4 -weak bisection. Accordingly, a counterexample for Conjecture 9 should be looked for among cubic graphs $G$ with $\phi_{c}(G) \geq 5$. When restricted to bridgeless graphs, there are several known simple schemas to recursively construct infinitely many cubic graphs, 2- and 3-connected, with circular flow number 5 , from smaller ones. We checked several such graphs, obtained from the Petersen graph, and found them to admit 4-weak bisections, but we can offer nothing in the direction of a general proof. We recently [4] developed an arsenal of construction methods which yield a rich variety of snarks (cyclically 4-edge-connected cubic graphs of girth at least 5 which admit no 4-CNZF) $G$ with $\phi_{c}(G) \geq 5$. Conjecture 9, as well as its restriction to bridgeless cubic graphs, seems, at least for now, far beyond our reach.

The vertices of any cubic graph can be partitioned into two color classes with no monochromatic component on more than two vertices, by repeatedly switching the color of a vertex which shares its color with more than one of its three neighbors. The resulting partition is not always a bisection, as $\left|V_{1}\right|=\left|V_{2}\right|$ is not guaranteed. However, the Petersen graph as well as $T_{k}$ for every $k \geq 0$ admit such a partition where $\left|V_{1}\right|-\left|V_{2}\right|=2$. This observation leads to the following:

Conjecture 10. The vertex set of any cubic graph can be partitioned into two color classes $V_{1}$ and $V_{2}$, satisfying ||$V_{1}|-| V_{2}|| \leq 2$, such that each monochromatic component contains at most two vertices.

Unlike nowhere-zero flows and $r$-strong bisections, the existence of $k$ - 
weak bisections is not restricted to bridgeless graphs. Hence, the existence of a 6-weak bisection in every cubic graph cannot be directly deduced from Seymour's 6-flow Theorem [8]. A stronger result however holds, as we prove in the following section.

\section{5-weak bisections}

As noted in the previous section, a $k$-weak bisection can be considered as a relaxed local variant of $k$-NZF, at least when referring to 2-connected cubic graphs. When comparing our results to what is known about strong bisections ( $k$-NZF's), we get the flavor of "One step down": Tutte's 5-flow conjecture asserts the existence of a 5-strong bisection for every 2-connected cubic graph. In Conjecture 9 (with the exception of the Petersen graph), 5 -strong bisections are replaced by 4 -weak bisections. With the following theorem, not only we replace "6-strong bisections" in Seymour's 6-flow theorem (when stated, without any loss of generality, for cubic graphs), by "5-weak bisections", but we also manage to do so for all cubic graphs, not necessarily bridgeless. Theorem 11 can be viewed as a local 5-flow theorem (although its restriction to bridgeless graphs happens to be the easier case).

Theorem 11. Every cubic graph admits a 5-weak bisection.

Our proof of Theorem 11 goes through a sequence of preparatory technical claims.

Definition 12. A valid factor $\mathcal{P}$ of a graph $G$ is a spanning subgraph of $G$, where every connected component is either a cycle, or a path, with the property that an odd (number of vertices) path of $\mathcal{P}$ is not an isolated vertex and its two endpoints are non-adjacent in $G$.

We first observe that a valid factor indeed exists in every cubic graph $G$ : By Vizing's theorem, $G$ has a (proper) 4-edge-coloring. Select all the edges of $G$ colored 1 or 2 . The subgraph obtained is a spanning subgraph whose connected components are paths and (even) cycles, and none of them is an isolated vertex. Now insert every edge of $G$ which connects the two endpoints of an odd path of the factor, to turn it into a cycle. The spanning subgraph obtained that way is a valid factor of $G$. 
Let $\mathcal{P}^{*}$ be a valid factor of $G$ such that the number of components of $\mathcal{P}^{*}$ is minimal, and, subject to that condition, the number of odd cycles of $\mathcal{P}^{*}$ is minimal.

Claim 13. We say that a vertex of $G$ is external, if it is the endpoint of a path of $\mathcal{P}^{*}$, or it belongs to an odd cycle of $\mathcal{P}^{*}$.

There is no edge in $G$ which connects two external vertices on two distinct components of $\mathcal{P}^{*}$.

Proof. Let $v_{1} v_{2}$ be an edge of $G$, where $v_{1}$ and $v_{2}$ are external and belong, respectively, to $T_{1}$ and $T_{2}$, two distinct components of $\mathcal{P}^{*}$. The number of components of $\mathcal{P}^{*}$ is decreased by one when the edge $v_{1} v_{2}$ is inserted to merge $T_{1}$ and $T_{2}$ into a single component $T$. If $T_{1}$ and $T_{2}$ are both paths, then so is $T$ (see Figure 3, left). Otherwise, if $T_{i}$, for $i=1$, or $i=2$, or both, is an odd cycle, then remove an edge $v_{i} x_{i}$, incident with $v_{i}$ in $T_{i}$, to turn $T$ into a path (see Figure 3, center and right). Finally, if the new path is odd and the two endpoints are adjacent, insert the edge which connects its endpoints to turn it into an odd cycle. Either way, we obtain a valid factor $\mathcal{Q}^{*}$ with one less component than $\mathcal{P}^{*}$, which contradicts the minimality of $\mathcal{P}^{*}$.
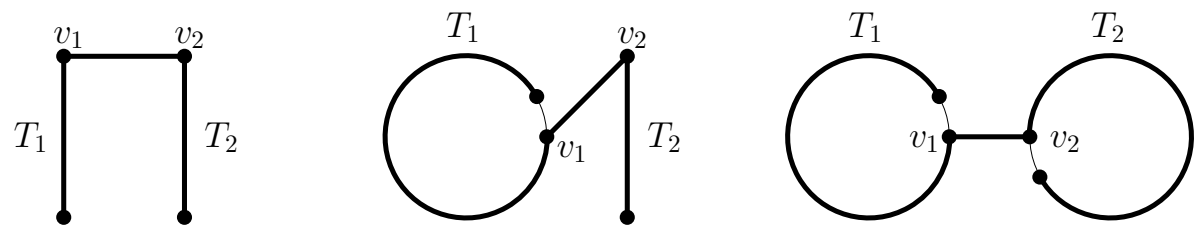

Figure 3: Three instances of Claim 13

Claim 14. Each odd cycle of $\mathcal{P}^{*}$ is chordless.

Proof. Assume that an odd cycle $C$ of $\mathcal{P}^{*}$ admits a chord. Since $C$ is odd and $G$ is cubic, not every vertex on $C$ belongs to a chord. We can then select a chord $u v$ of $C$ and a vertex $w$, adjacent to $u$ on $C$, such that $w$ does not belong to a chord of $C$. Let $x$ be the neighbor of $v$ on $C$ lying on the portion of $C$ between $v$ and $u$ which avoids $w$. We now replace $C$ in $\mathcal{P}^{*}$ by the path $(C \cup u v) \backslash\{x v, u w\}$ (see Figure 4, left), As $w$ belongs to no chord of $C$, the endpoints $w$ and $x$ of this new (odd) path are non-adjacent, so the obtained factor $\mathcal{Q}^{*}$ is indeed valid, contains the same number of components as $\mathcal{P}^{*}$, and has less odd cycles than $\mathcal{P}^{*}$. This contradicts the minimality of $\mathcal{P}^{*}$. 

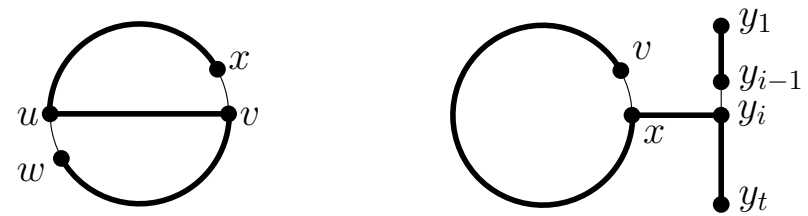

Figure 4: Claim 14 (left) and Claim 15 (right).

Claim 15. Assume that $G$ contains an edge $x y_{i}$ such that $x$ is on an odd cycle $C$ of $\mathcal{P}^{*}$ and $y_{i}$ is a vertex of a path $P=y_{1} y_{2} \cdots y_{t}$ of $\mathcal{P}^{*}$. Then $i$ is even, $t$ is odd, and if $i \neq 2$ then $y_{1} y_{i-1}$ is an edge of $G$ and if $i \neq t-1$ then $y_{i+1} y_{t}$ is an edge of $G$.

Proof. Let $v$ be a neighbor of $x$ on $C$. If $t$ is even, or if $t$ and $i$ are both odd, then at least one of the paths $y_{1} y_{2} \cdots y_{i-1}$ and $y_{i+1} y_{i+2} \cdots y_{t}$ contains an even number of vertices. Assume then that $i-1$ is even (otherwise reverse the labeling $1,2, \ldots, t$ on $P$ ). Let $\mathcal{Q}^{*}$ be obtained from $\mathcal{P}^{*}$ by replacing $C$ and $P$ by (1) the path which is the union of $C \backslash v x$, the edge $x y_{i}$, and the path $y_{i+1} y_{i+2} \cdots y_{t}$ and (2) the even path $y_{1} y_{2} \cdots y_{i-1}$ (see Figure 4, right). By Claim 13, $v y_{t}$ is not an edge of $G$ and therefore $\mathcal{Q}^{*}$ is indeed a valid factor. $\mathcal{Q}^{*}$ contains the same number of components as $\mathcal{P}^{*}$ but has one odd cycle less, a contradiction.

The contradiction above implies that $t$ is odd and $i$ is even. So we assume now that this is indeed the case, but $i \neq 2$ and $y_{1}$ and $y_{i-1}$ are nonadjacent. We now construct $\mathcal{Q}^{*}$ exactly as we did before. This time the path $y_{1} y_{2} \cdots y_{i-1}$ is odd, however, its endpoints $y_{1}$ and $y_{i-1}$ are non-adjacent, and $i \neq 2$ guarantees that it is not an isolated vertex. Consequently, $\mathcal{Q}^{*}$ is a valid factor also in this case and the same contradiction still holds. The case where $i=t-1$ and $y_{t}$ is non-adjacent to $y_{i+1}$ is handled identically, after reversing the labeling along $P$.

Claim 16. Every two vertices on an odd cycle of $\mathcal{P}^{*}$ are connected to two distinct odd paths of $\mathcal{P}^{*}$.

Proof. Let $C$ be an odd cycle of $\mathcal{P}^{*}$. By Claim 14, $C$ is chordless, so every vertex of $C$ is adjacent with one vertex on another component of $\mathcal{P}^{*}$. By Claims 13 and 15, that other component is necessarily an odd path. Assume now to the contrary, that $P=y_{1} y_{2} \cdots y_{t}$ is a path of $\mathcal{P}^{*}$ and that $G$ contains two edges $x y_{i}$ and $z y_{j}$, with $i<j$. By Claim 15, $i$ and $j$ are even, and therefore $i \leq j-2$. It follows that $j \neq 2$ and $i \neq t-1$ and so by Claim 15 , 
$G$ contains the two edges $y_{1} y_{j-1}$ and $y_{i+1} y_{t}$. Let $v$ be a neighbor of $x$ on $C$ (say $v \neq z$, although this is not really essential). Let $\mathcal{Q}^{*}$ be obtained from $\mathcal{P}^{*}$ by replacing $C$ and $P$ by the path $\left(C \cup P \cup\left\{x y_{i}, y_{1} y_{j-1}, y_{i+1} y_{t}\right\}\right) \backslash$ $\left\{x v, y_{i} y_{i+1}, y_{j-1} y_{j}\right\}$ (see Figure 5, left). $C$ and $P$ are both odd, so the new merged path is even and $\mathcal{Q}^{*}$ is a valid factor with one component less than $\mathcal{P}^{*}$, a contradiction.
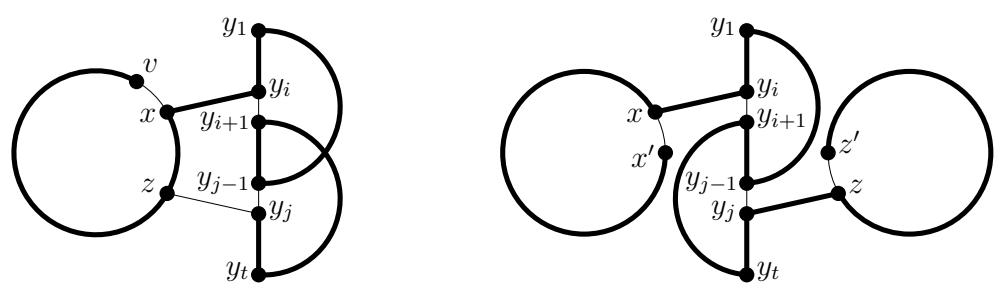

Figure 5: Claim 16 (left) and Claim 17 (right).

Claim 17. A path of $\mathcal{P}^{*}$ cannot be connected to two distinct odd cycles of $\mathcal{P}^{*}$.

Proof. Assume that $\mathcal{P}^{*}$ contains a path $P=y_{1} y_{2} \cdots y_{t}$ and two odd cycles $C$ and $C^{\prime}$, such that $G$ contains two edges $x y_{i}$ and $z y_{j}$ with $i<j, x \in C$, and $z \in C^{\prime}$. By Claim 15, $i$ and $j$ are even, and therefore $i \leq j-2$. It follows that $j \neq 2$ and $i \neq t-1$, so by Claim 15, $G$ contains the two edges $y_{1} y_{j-1}$ and $y_{i+1} y_{t}$. Let $x^{\prime}$ be a neighbor of $x$ on $C$ and $z^{\prime}$ a neighbor of $z$ on $C^{\prime}$. Let $\mathcal{Q}^{*}$ be obtained from $\mathcal{P}^{*}$ by removing $P, C$ and $C^{\prime}$ and inserting the path

$$
\left(C \cup C^{\prime} \cup P \cup\left\{x y_{i}, y_{1} y_{j-1}, y_{i+1} y_{t}, y_{i} z\right\}\right) \backslash\left\{x x^{\prime}, y_{i} y_{i+1}, y_{j-1} y_{j}, z z^{\prime}\right\},
$$

see Figure 5 (right). By Claim 13 the endpoints of the new path, $x^{\prime}$ and $z^{\prime}$ are non-adjacent and $\mathcal{Q}^{*}$ is indeed a valid factor with two components less than $\mathcal{P}^{*}$, a contradiction.

Claim 18. The number of odd paths of $\mathcal{P}^{*}$ is at least three times the number of odd cycles of $\mathcal{P}^{*}$.

Proof. Claims 16 and 17 establish an injective function from the set of all vertices on odd cycles of $\mathcal{P}^{*}$, into the set of odd paths of $\mathcal{P}^{*}$. Since there are at least three vertices on each cycle, the assertion of the claim immediately follows. 
We are now set to complete the proof of Theorem 11 .

Let $G$ be a cubic graph and let $\mathcal{P}^{*}$ be the valid factor defined above. As every component of $\mathcal{P}^{*}$ is either a path or a cycle, we now assign the colors 1 and 2 , alternately to vertices along every component and define the set $V_{i}$, $i=1,2$ as the set of all vertices with the color $i$. This coloring schema is not uniquely defined and leaves room for further refinement. Let us start with the odd cycles. On every odd cycle $C$ an alternating coloring leaves two adjacent vertices $x$ and $z$ with the same color, and $x z$ is the only edge of $C$ with the same color on its two endvertices. We define $x$ to be the first vertex of $C$ and $z$ the last one. Now let $x$ be the first vertex of an odd cycle $C$ and let $P$ be the odd path, to which $x$ is connected, according to Claim 16, by an edge $x y$. We now assign a color to $y$ such that $x$ and $y$ have distinct colors and then proceed from $y$ to alternately color all vertices along $P$ in both directions. Note that by Claim 17, any path of $\mathcal{P}^{*}$ is connected to at most one odd cycle of $\mathcal{P}^{*}$, so the coloring above is well-defined. For $\left(V_{1}, V_{2}\right)$ to be a bisection we should keep the numbers of vertices colored 1 and colored 2 equal. The alternating coloring schema guarantees this equality on every even component. Among the vertices of an odd component, be it an odd cycle, or an odd path, the color of the first (and the last) vertex gains a majority of one. So far we determined the coloring of all odd cycles and one odd path against every odd cycle. Let $n$ be the number of odd cycles. The advantage of one color over the other, so far, is at most $2 n$. By Claim 18 there are still at least that many odd paths not yet colored. Each of them can change the difference between $\left|V_{1}\right|$ and $\left|V_{2}\right|$ by one, in any direction, by selecting the color of its first vertex. That, plus the fact that the total number of odd components is even, makes it possible to complete the coloring with $\left|V_{1}\right|=\left|V_{2}\right|$, as required. An example of coloring obtained is depicted in Figure 6 .
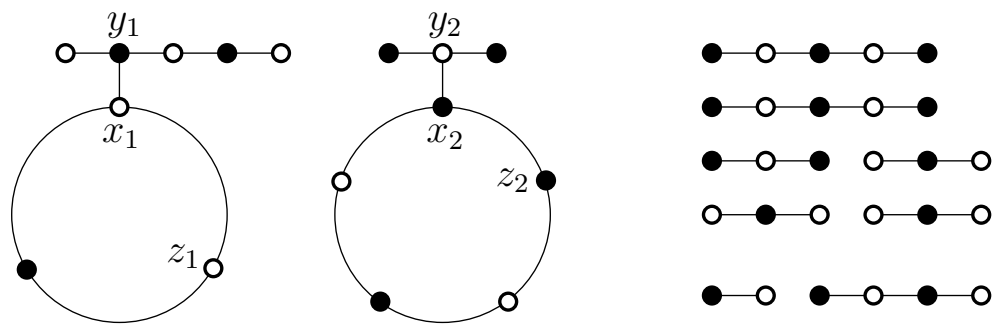

Figure 6: A typical 2-coloring of $\mathcal{P}^{*}$. 
We now prove that there is no monochromatic component on more than three vertices: Since no component of $\mathcal{P}^{*}$ is an isolated vertex, our alternating coloring schema provides, for every vertex, at least one neighbor of the opposite color. That observation eliminates monochromatic $K_{1,3}$. It remains to consider monochromatic copies of $P_{4}$ (the path on 4 vertices) and $K_{3}$ (the triangle). We say that a vertex $x$ is good, if at least two of its three neighbors differ from $x$ in color; $x$ is bad if it shares his color with two of its neighbors. A monochromatic $P_{4}$ or $K_{3}$ contains two adjacent bad vertices of the same color. So, we only need to show that no two such vertices are adjacent. Every inner (not an endpoint) vertex of a path is necessarily good. The only bad vertices on cycles may be the last vertices of the odd cycles. Notice that the first vertex $x$ of an odd cycle $C$ differs in color from one of its neighbors on $C$ (not the last one), and from its neighbor $y$ on an odd path $P$, so $x$ is a good vertex. It turns out then, that all bad vertices, if there are any, are external (as defined in Claim 13). Therefore, by Claim 13 , no two bad vertices from two distinct components of $\mathcal{P}^{*}$ are adjacent. By the definition of a valid factor, the two endpoints of an odd path are non-adjacent; An odd cycle has at most one bad vertex (its last vertex) and the two endpoints of an even path are of distinct colors. In conclusion, there is no monochromatic $P_{4}$ or $K_{3}$, so $\left(V_{1}, V_{2}\right)$ is indeed a 5 -weak bisection.

As already mentioned, Theorem 11 can be looked at, as a (much) weaker version of the assertion of Tutte's 5-Flow Conjecture. Not precisely weaker, because Theorem 11 holds for every cubic graph, while the 5-Flow Conjecture relates only to bridgeless graphs. When searching potential directions to deal with nowhere-zero flow problems, it is worth noting that we can suggest a very short and simple proof of Theorem 11, when restricted to cubic graphs which admits perfect matchings, bridgeless graphs in particular. A similar proof appears in [10], although the result is not explicitly stated there.

Proof. Let $G=(V, E)$, be a cubic graph and $M$ a perfect matching in $G$. Then $T=E \backslash M$ is a 2-factor (a union of disjoint simple cycles). Let $S$ be a set of edges, which consists of two consecutive edges from each odd cycle of $T$. Accordingly, $T^{\prime}=T \backslash S$ is a union of disjoint even cycles and even (odd number of edges) paths and, as such, can be partitioned into two matchings $T_{1}$ and $T_{2}$. We make sure to include in $T_{1}$ the two end-edges of each path of $T^{\prime}$. That way, $S$ and $T_{2}$ have no common vertex, so every connected component of $S \cup T_{2}$ is either a single edge of $T_{2}$, or two edges of 
$S$. As the union of two matchings, $P=M \cup T_{1}$ is bipartite. Let $\left(V_{1}, V_{2}\right)$ be any bipartition of $P$. Since $M$ is a perfect matching, $\left(V_{1}, V_{2}\right)$ is a bisection. An edge of $P$ connects two vertices of distinct colors, so all monochromatic edges belong to $E \backslash P=S \cup T_{2}$. Therefore there are at most two edges in a monochromatic component and $\left(V_{1}, V_{2}\right)$ is indeed a 5 -weak bisection.

Let us add that, following this proof, the number of monochromatic paths on 3 vertices, can be bounded by the oddness (minimum number of odd components in a 2-factor) of $G$.

\section{References}

[1] A. Ban and N. Linial, Private communication.

[2] A. Ban and N. Linial, Internal Partitions of Regular Graphs, J. Graph Theory. doi:10.1002/jgt.21909

[3] A. Bondy and P. Hell, A note on the star chromatic number, J. Graph Theory 14(4) (1990), 479-482.

[4] L. Esperet, G. Mazzuoccolo and M. Tarsi The structure of graphs with circular flow number 5 or more, and the complexity of their recognition problem J. Comb. 7(2) (2016), 453-479.

[5] L.A. Goddyn, M. Tarsi, and C.Q. Zhang, On $(k, d)$-colorings and fractional nowhere-zero flows, J. Graph Theory 28(3) (1998), 155-161.

[6] F. Jaeger, Balanced Valuations and Flows in Multigraphs, Proc. Amer. Math Soc. 55(1) (1976), 237-242.

[7] J. Petersen, Die Theorie der regulären graphs, Acta Math. 15 (1891), $193-220$.

[8] P.D. Seymour, Nowhere-zero 6-flows, J. Combin. Theory Ser. B 30(2) (1981), 130-135.

[9] E. Steffen, Circular flow numbers of regular multigraphs, J. Graph Theory $\mathbf{3 6 ( 1 )}$ (2001), 24-34.

[10] E. Steffen, Tutte's 5-flow conjecture for highly cyclically connected cubic graphs, Discrete Math. 310 (2010), 385-389. 
[11] W.T. Tutte, A contribution to the theory of chromatic polynomials, Canad. J. Math. 6 (1954), 80-91. 